\title{
Upper airway.4: Sarcoidosis of the upper respiratory tract (SURT)
}

\author{
Robert P Baughman, ${ }^{1}$ Elyse E Lower, ${ }^{2}$ Thomas Tami ${ }^{3}$
}

${ }^{1}$ University of Cincinnati Medical Center, Cincinnati, Ohio, USA

${ }^{2}$ Oncology Hematolgy Care, Cincinnati, Ohio, USA ${ }^{3}$ Cincinnati Sinus Institute, Cincinnati, Ohio, USA

\section{Correspondence to}

Professor Robert P Baughman, 1001 Holmes, Eden Ave, Cincinnati, OH 45267-0565, USA; bob.baughman@uc.edu

Received 5 October 2009 Accepted 27 November 2009

\section{ABSTRACT}

Sarcoidosis of the upper respiratory tract (SURT) includes nasopharyngeal, laryngeal and tracheal disease. Documented SURT occurs in $\sim 5 \%$ of patients, although upper airway symptoms are more common in patients with sarcoidosis. Wegener granulomatosis may have a similar appearance to SURT, but there are important differences in the manifestations of the disease locally as well as systemically. In some cases, topical treatment is adequate to control SURT. However, many patients with significant sinus or laryngeal disease require long-term systemic treatment. While corticosteroids are often the initial drug of choice, there have been several reports of the effectiveness of cytotoxic agents and anti-tumour necrosis factor antibodies.

Upper airway disease can be a manifestation of sarcoidosis. Although the lung and mediastinum are more usual locations, the granulomas of sarcoidosis can affect the entire respiratory tree including the upper airway. The purpose of this review is to discuss the clinical presentation, method of diagnosis and treatment for upper airway sarcoidosis. Local treatment may provide some temporary symptomatic relief; however, long-term systemic anti-inflammatory treatment is often required. Recent advances in the treatment of sarcoidosis have led to new therapeutic options for these frequently refractory conditions.

\section{CLINICAL MANIFESTATIONS}

Biopsy-confirmed sarcoidosis of the upper respiratory tract (SURT) occurs in $\sim 2-3 \%$ of patients with sarcoidosis. ${ }^{12}$ Nasal symptoms are frequently reported. In a prospective study of 159 consecutive patients with sarcoidosis followed in one clinic, 27 patients reported upper airway symptoms unresponsive to 3 weeks of topical steroids and/or antibiotics. All 27 patients underwent sinus CT scanning and subsequently five patients $3 \%$ of total) had biopsy confirmation of SURT. ${ }^{1}$

Nasal stuffiness, blockage, crusting and anosomia are frequently noted at initial presentation. ${ }^{2} 3$ Less common are epistaxis, facial pain and dysphagia. Destruction of nasal sinuses is a feature of more chronic disease. ${ }^{1}$ Erythematous nodules on the turbinates and septum, sometimes called granulations, are frequently identified. ${ }^{4}$ Less frequent is saddle nose deformity and septal perforation.

Sinus disease can occur as a consequence of direct sinus extension or from extrinsic compression. Orbital lesions can cause not only diplopia, but also sinus congestion and dacrocystitis, a complication secondary to the granulomas of sarcoidosis leading to poor clearance of tears. When identified, this can be corrected surgically.

While nasal disease is the most common form of SURT, all parts of the upper respiratory tract can be affected. Laryngeal involvement can create total airway obstruction leading to respiratory arrest. In those patients, a tracheostomy may be the only way to relieve obstruction immediately. ${ }^{5}$ The clinical manifestations of SURT are diverse because any area can be involved with granulomas. Voice change may be the first manifestation of laryngeal disease. ${ }^{5}$ While laryngeal disease can be associated with sinonasal disease, ${ }^{46}$ a patient can present with only laryngeal or pharyngeal disease. ${ }^{1} 24$

Figure 1 reveals the CT scan of a patient with sarcoidosis who originally presented with laryngeal sarcoidosis. As can be seen, the right upper lobe is narrowed (circle) with subsequent collapse of the distal airway. In this case, endobronchial sarcoidosis created airway narrowing. While endoluminal stenosis of the proximal airways is associated with SURT, $^{7}$ it can also develop independently of sinonasal disease. $^{8}$

The clinical features of endobronchial sarcoidosis vary depending on the degree of airway narrowing. Endobronchial disease is fairly common in sarcoidosis. In a prospective study of 34 biopsy-confirmed patients undergoing diagnostic bronchoscopy, endobronchial abnormalities including erythema and/or mucosal thickening (figure 2) were visualised in $71 \%$ of them. Overall, endobronchial biopsies demonstrated granulomas in 21 of 34 (61\%) patients. A positive biopsy for non-caseating granulomas was more likely to be encountered in patients with an abnormal airway. ${ }^{9}$ Endobronchial disease is frequently associated with cough.

More severe narrowing can lead to near total obstruction of the airways. Patients with significant airway narrowing often experience wheezing, squeaks and even stridor if the airway narrowing involves the extrathoracic airway. ${ }^{78}$ Wheezing may be fixed and not changed by cough or bronchodilators. These changes can be associated with an obstructive disease pattern on pulmonary function testing $^{8}$ with no reversibility with bronchodilators. ${ }^{7}$ In one series of 18 patients with proximal airway stenosis, Chambellan et al identified a single area of stenosis in only $3(16 \%)$ cases. While diffuse airway narrowing was identified in some patients, multiple stenotic sites were encountered in two-thirds of their patients. As noted in figure 1, occasionally a CT scan identified adenopathy associated with airway narrowing. However, not extrinsic compression, but endobronchial narrowing with erythema and mucosal changes was considered causative of the constriction in most cases. ${ }^{7}$ High 


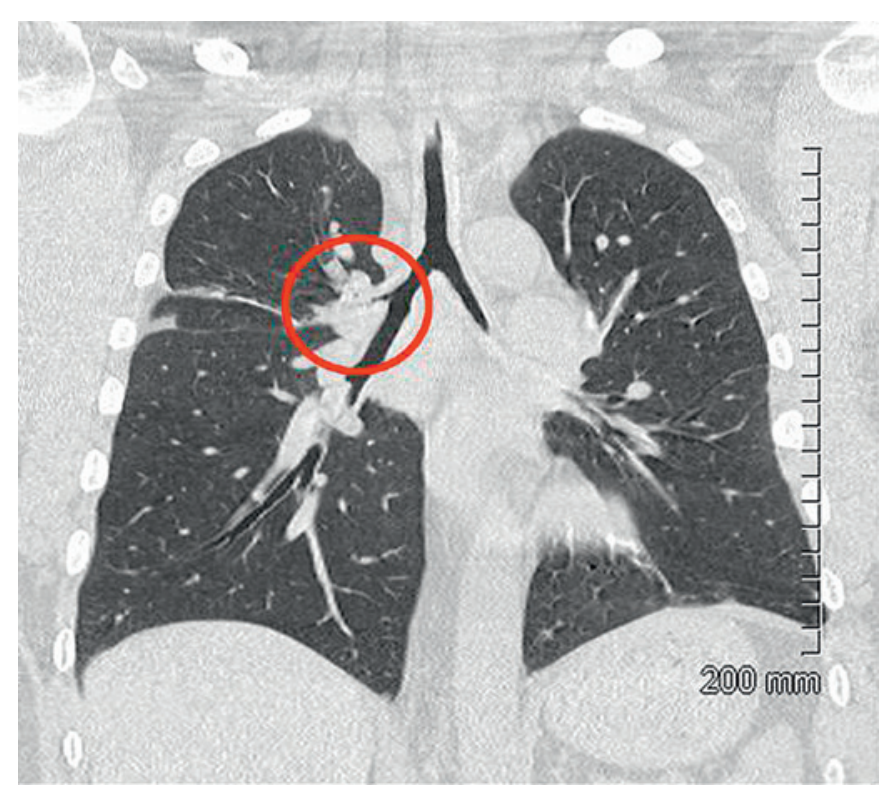

Figure 1 A patient with a past history of laryngeal sarcoidosis. They now have a fixed wheeze. A coronal view of the trachea and major airways demonstrates narrowing of the right upper lobe (circle) with associated atelectasis of the anterior segment of the right upper lobe. Figure courtesy of Dr Robert Dales.

resolution CT scanning may be a useful adjunct to bronchoscopy in identifying airway disease. ${ }^{10}$

Sarcoidosis is a multiorgan disease, with pulmonary involvement found in $>90 \%$ of cases. Although skin lesions are identified in $20-30 \%$ of patients with general sarcoidosis, facial disease is reported in $<10 \%$ of patients. In contrast, facial skin lesions are common in patients with SURT, including lupus pernio, which is a specific facial manifestation of chronic sarcoidosis. This cutaneous finding is highly associated with sinus disease. ${ }^{6}$ In one series $\sim 50 \%$ of patients with lupus pernio also experienced sinus disease. ${ }^{11}$ Likewise, in another series of patients with SURT, lupus pernio was identified in half of the cases. ${ }^{3}$ Sinus destruction has been reported as a manifestation of sarcoidosis. ${ }^{12}$

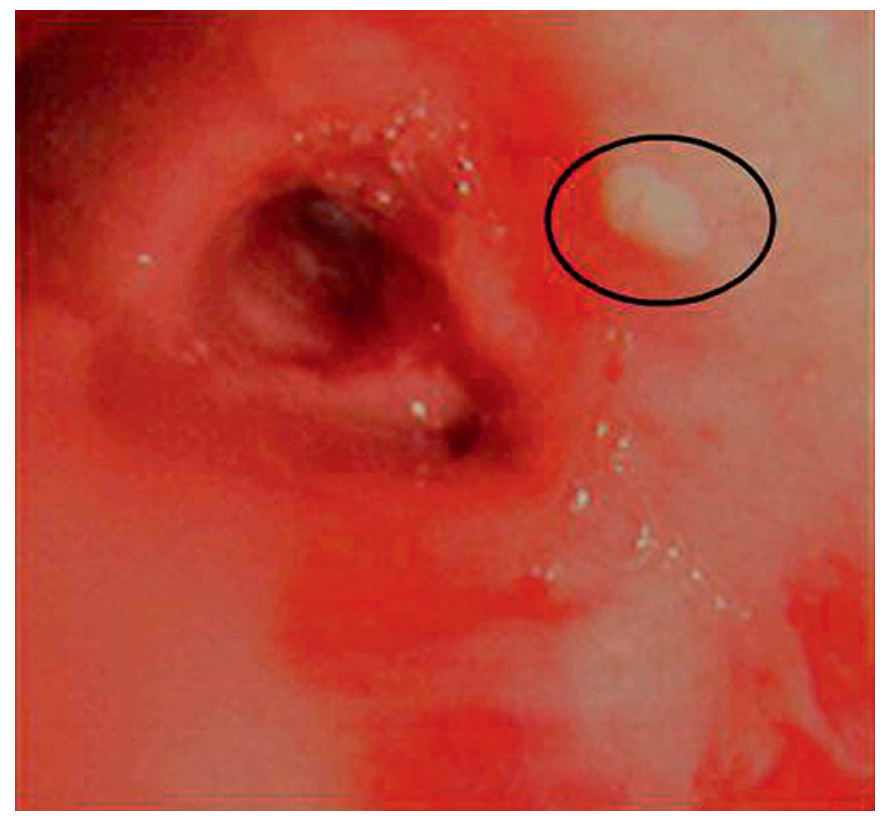

Figure 2 Airway oedema, mucosal hypertrophy and nodule (circled) in a patient with endobronchial sarcoidosis.
A subset of sarcoidosis patients may present with Heerfordt syndrome, a complex of fever, uveitis, parotid gland swelling and facial nerve palsy. ${ }^{13}$ Parotid disease can also occur independently of uveitis and facial nerve paralysis. Subsequent dry mouth can be a consequence of parotid involvement. Because of frequent lacrimal gland involvement, patients with sarcoidosis can develop secondary Sjogren syndrome from their disease.

\section{DIAGNOSIS}

The aetiology of sarcoidosis remains elusive, hence the diagnosis remains one of exclusion. ${ }^{14}$ The current guidelines define sarcoidosis with a compatible clinical presentation and biopsy confirmation of granulomatous disease with other causes of granulomatous reaction excluded. Certain manifestations of the disease are highly supportive of the diagnosis, including the presence of lupus pernio and Lofgren and Heerfordt syndrome. ${ }^{14}$ For specific organ involvement, criteria for definite and probable disease have been proposed. Because of the multiorgan nature of sarcoidosis, all patients with possible sarcoidosis should undergo routine evaluation for potential involvement of other organs. Specific information should be solicited regarding pulmonary, skin, eye, cardiac and neurological symptoms. Also a chest roentgenogram, spirometry, and serum for liver function, calcium and a complete blood count should be obtained. Information from these tests may prove useful in confirming the diagnosis of sarcoidosis as well as possibly identifying indications for treatment.

Two groups have suggested specific criteria for the diagnosis of sinus sarcoidosis. ${ }^{45}$ Table 1 highlights the similarities and differences between the two proposals. Because symptoms are often non-specific, both groups emphasise the importance of radiology in identifying sinus disease. Diffuse disease involving multiple sinuses is common (figure 3). If possible, a biopsy should be performed to confirm the presence of granulomatous inflammation, and the specimen should undergo special studies for fungus or mycobacteria as the cause of the granulomatous reaction. Ancillary blood tests should include an ACE level and antineutrophil cytoplasmic antibody (ANCA). The gallium scan can often demonstrate increased activity in the sinuses of untreated patients with sarcoidosis, ${ }^{4}$ with the "Panda" sign demonstrating activity in both parotid glands and eyes of a patient with active disease. ${ }^{14}$ However, gallium scanning is not specific for sarcoidosis as it may also be positive in active Wegener granulomatosis. ${ }^{16}$ Table 1 emphasises the importance of excluding other conditions that can mimic sarcoidosis.

\section{SURT VERSUS WEGENER GRANULOMATOSIS: ORGAN INVOLVEMENT}

Wegener granulomatosis is an important diagnostic consideration for a patient with granulomatous disease involving the nose, paranasal sinuses or upper airways. ${ }^{17}$ Table 2 summarises some features that are useful in distinguishing between Wegener granulomatosis and sarcoidosis. ${ }^{18}$ Although destruction of the upper airway is commonly associated with Wegener disease, these changes can also be seen with sarcoidosis. ${ }^{19}$ Although uveitis is the most common manifestation of sarcoid eye disease, patients can also develop optic neuritis and corneal deposits termed "mutton fat". Eye involvement is more frequent in Wegener granulomatosis than sarcoidosis, with some series reporting $\sim 50 \%$ of patients with Wegener granulomatosis developing some form of eye disease. ${ }^{20}$ Cutaneous disease can be 
Table 1 Diagnostic criteria for sarcoidosis sinus disease

\begin{tabular}{|c|c|c|}
\hline & deShazo et al ${ }^{15}$ & Braun et $a I^{4}$ \\
\hline History & & $\begin{array}{l}\text { Chronic rhinosinusitis } \\
\text { poorly responsive to } \\
\text { conventional treatment }\end{array}$ \\
\hline $\begin{array}{l}\text { Radiology/ } \\
\text { examination }\end{array}$ & $\begin{array}{l}\text { Radiological evidence of } \\
\text { sinusitis }\end{array}$ & $\begin{array}{l}\text { Radiological evidence of } \\
\text { rhinosinusitis }\end{array}$ \\
\hline Pathology & $\begin{array}{l}\text { Histopathological confirmation } \\
\text { of non-caseating granuloma } \\
\text { in the sinus tissue supported } \\
\text { by negative stains for fungus } \\
\text { and acid-fast bacilli }\end{array}$ & $\begin{array}{l}\text { Histopathological confirmation } \\
\text { of non-caseating granuloma }\end{array}$ \\
\hline $\begin{array}{l}\text { Ancillary blood } \\
\text { tests }\end{array}$ & $\begin{array}{l}\text { Negative serological test } \\
\text { results for syphilis and } \\
\text { antineutrophil cytoplasmic } \\
\text { antibodies }\end{array}$ & Elevated ACE \\
\hline Other testing & & $\begin{array}{l}\text { Positive gallium scan } \\
\text { (if performed) }\end{array}$ \\
\hline $\begin{array}{l}\text { Exclusion } \\
\text { criteria }\end{array}$ & $\begin{array}{l}\text { No clinical evidence of other } \\
\text { processes associated with } \\
\text { granulomatous nasal and } \\
\text { sinus inflammation }\end{array}$ & $\begin{array}{l}\text { No evidence of other } \\
\text { granulomatous diseases, such } \\
\text { as Wegener granulomatosis }\end{array}$ \\
\hline $\begin{array}{l}\text { Supportive } \\
\text { information }\end{array}$ & & $\begin{array}{l}\text { Frequent evidence of systemic } \\
\text { sarcoidosis, especially pulmonary }\end{array}$ \\
\hline
\end{tabular}

identified in either sarcoidosis or Wegener granulomatosis. However, the maculopapular skin lesions of sarcoidosis, including those of lupus pernio, differ from the leucocytoclastic vasculitis or necrotic skin lesions identified with Wegener disease. ${ }^{21}$

Although lung involvement is frequently identified in both sarcoidosis and Wegener granulomatosis, the roentgenographic abnormalities differ. The lung disease which occurs in $>90 \%$ of patients with sarcoidosis can be categorised into four patterns using the Scadding classification system ${ }^{14}$ : stage 1 , hilar adenopathy alone; stage 2 , hilar adenopathy with pulmonary infiltrates; stage 3, pulmonary infiltrates alone; and stage 4, pulmonary fibrosis. As with most classification schemes, some staging reproducibility difficulties can be encountered for individual patients with sarcoidosis ${ }^{22}$; however, the overall appearance of the chest roentgenogram is quite useful in recognising sarcoidosis. CT scanning will often identify mediastinal adenopathy not suspected by chest roentgenogram, and lymph nodes $>15 \mathrm{~mm}$ in patients with diffuse infiltrates are probably due to sarcoidosis. ${ }^{23}$ In contrast, the chest roentgenogram and CT scan in Wegener granulomatosis often reveal lung masses and fleeting infiltrates. Cavitary masses are commonly seen in Wegener vasculitis but are rare in sarcoidosis. ${ }^{18}$

The kidneys can be affected in either sarcoidosis or Wegener granulomatosis. However, the renal disease identified in sarcoidosis is usually a consequence of hypercalcaemia or possibly granulomatous interstitial nephritis. ${ }^{14}$ Significant renal disease is a hallmark of extensive Wegener granulomatosis. Haematuria and proteinuria are commonly identified in patients with Wegener granulomatois, but rare in patients with sarcoidosis. Kidney biopsies from patients with Wegener disease will display focal necrotising glomerulitis. ${ }^{18}$

Although neurological disease is reported in $\sim 10 \%$ of patients with sarcoidosis, ${ }^{24} \sim 50 \%$ of patients with Wegener granulomatois have neurological lesions. ${ }^{25}$ In addition to differences in frequency, the pattern of neurological involvement varies between these two conditions. Patients with sarcoidosis may experience either central nervous system (CNS) or peripheral nervous system disease. MRI with gadolinium remains the radiographic procedure of choice for CNS disease secondary to sarcoidosis. Although diverse abnormalities have been reported, mass lesions and meningeal enhancement remain common
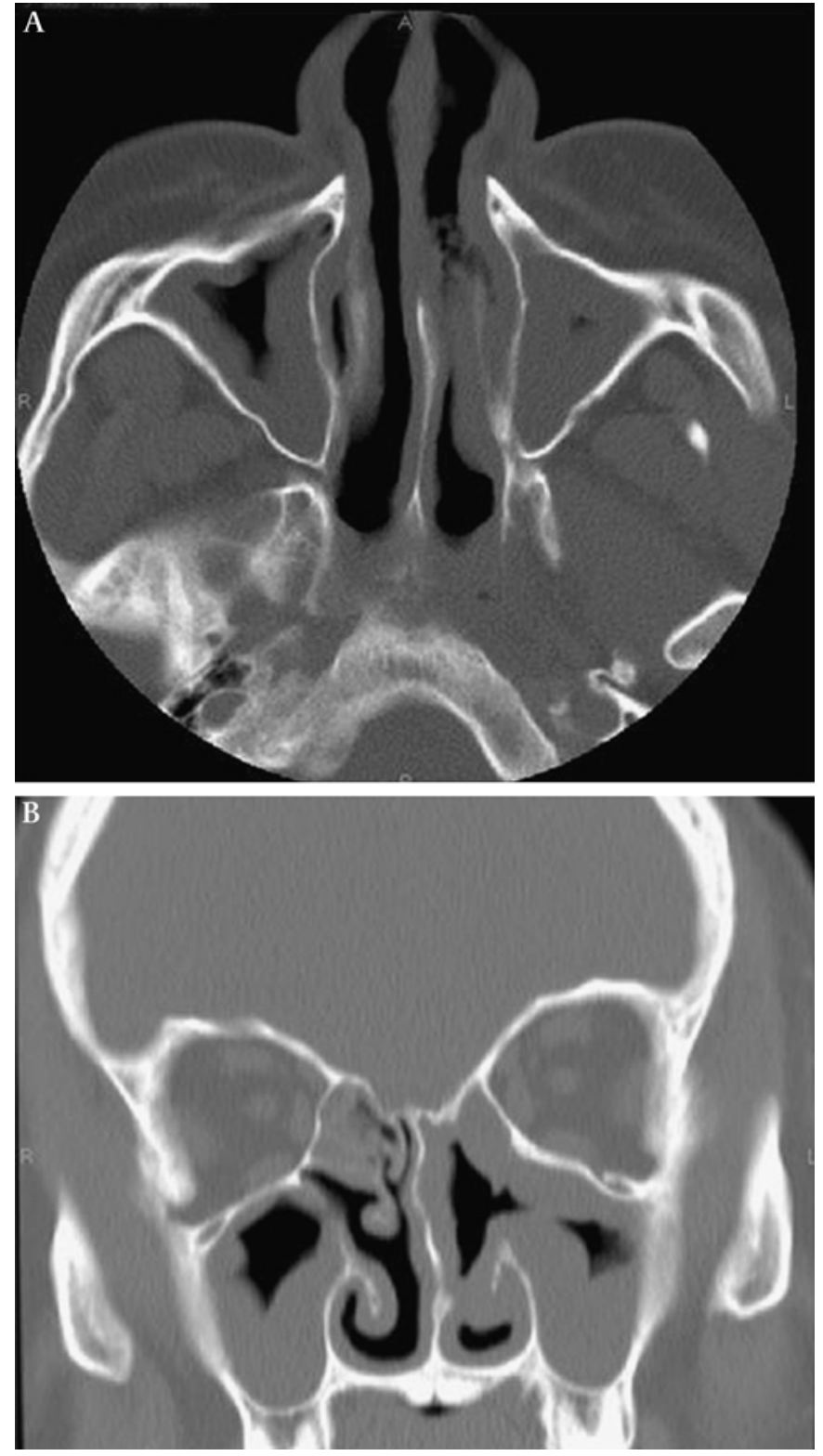

Figure 3 CT scan sagittal $(A)$ and coronal $(B)$ images demonstrating diffuse sinus involvement from biopsy-confirmed sarcoidosis.Airway oedema, mucosal hypertrophy and nodule (circled) in a patient with endobronchial sarcoidosis.

findings. ${ }^{24}$ Direct bone involvement with associated specific radiographic features, including cysts, can be seen in spinal sarcoidosis. These findings may assist in the diagnosis of sarcoidosis. In contrast, peripheral neuropathy is the most common finding in Wegener disease, ${ }^{25}$ with the CNS lesions usually the result of vasculitis.

As previously discussed, involvement of the proximal airway with stenosis can be seen in some cases of sarcoidosis. ${ }^{7} 8$ However, this is a rare finding, with only 18 of 2500 patients identified at one institution. ${ }^{7}$ On the other hand, airway disease in Wegener granulomatosis is more common. In one study of 51 consecutive cases, endobronchial abnormalities were identified in $>50 \%$ of cases including subglottic stenosis, ulcerating tracheobronchitis with or without inflammatory pseudotumours (60\%), and tracheal and/or bronchial stenosis without inflammation. ${ }^{26}$ The airway lesions in Wegener granulomatosis tend to have more ulcerations and friability. 
Table 2 Comparison between sarcoidosis and Wegener granulomatosis

\begin{tabular}{lll}
\hline & Sarcoidosis & Wegener granulomatosis \\
\hline $\begin{array}{l}\text { Clinical presentation } \\
\text { Upper airway }\end{array}$ & Occurs in $<10 \%$ of cases & Extremely common \\
Lung & Common & Common \\
& Adenopathy & Nodular infiltrates, sometimes with cavities \\
Diffuse infiltrates, usually upper lobes & Infiltrates localised to areas of haemorrhage, often lower lobe \\
Eye & Uveitis, retinitis, optic neuritis & Episcleritis \\
Skin & Maculopapular lesions & Vasculitic lesions \\
& Lupus pernio & Occasionally seen \\
Kidney & Glomerulopnephritis rare, renal failure usually due to hypercalcaemia & Focal necrotising glomerulitis in over half of cases \\
Neurological disease & $10 \%$ of patients. Often mass seen on MRI & Up to $30 \%$ of cases will have lesions. Usually due to vasculitic lesions \\
Joints & Uncommon. Can see specific bone cysts & Uncommon \\
Tracheal/proximal airway stenosis & Rare & Common \\
ANCA & Negative & Positive in $>80 \%$ of cases \\
ACE & Positive in $>60 \%$ of new cases & Negative \\
Treatment & & \\
Corticosteroids & Drug of choice for initial management & Supportive but not used for maintenance therapy \\
Methotrexate & Effective & Effective \\
Azathioprine & Effective & Effective \\
Cyclophosphamide & Reserved for refractory cases & Treatment for extensive disease \\
Anti-TNF therapy & Effective for refractory disease & Not effective for most patients \\
Ritixumab & Effectiveness unknown & Effective for refractory cases
\end{tabular}

ANCA, antineutrophil cytoplasmic antibody; TNF, tumour necrosis factor.

\section{SURT VERSUS WEGNER GRANULOMATOSIS: LABORATORY TESTS}

Although imperfect, serum blood testing for ACE and ANCA should be performed in the evaluation of granulomatous disease of the upper airway. The ACE level can be increased in over half of patients with active sarcoidosis, including those with SURT. ${ }^{4}$ However, corticosteroid treatment can normalise the ACE level despite the disease remaining active. In addition, recent studies suggest that different polymorphisms of the ACE gene can be responsible for small changes in the serum ACE level. ${ }^{14}$ The most common reason for a low ACE level remains the concomitant use of an ACE inhibitor, which blocks the biological assay that is used to measure ACE levels.

Although the serum ANCA is positive in almost all patients with active severe Wegener disease, at least $20 \%$ of patients with active limited disease, including upper airway disease, will have negative ANCA serology. ${ }^{27}$ However, patients with SURT do not have a positive ANCA serology. ${ }^{3}$

Fungal and mycobacterial infection must also be considered in evaluating a patient with possible SURT. Pathology specimens should undergo special stains and cultures for these organisms. Bronchoscopy samples are quite useful in diagnosing both mycobacterial and fungal infections, although the diagnostic sensitivity is higher for mycobacterial infections. Mediastinal fibrosis secondary to infections such as histoplasmosis can lead to compression and airway obliteration. Because of the persistent inflammatory response despite antifungal treatment and other procedures, this condition can be very difficult to treat. Mediastinal fibrosis rarely occurs in sarcoidosis.

\section{TREATMENT}

The management of SURT may require local or systemic treatment depending on the severity of the condition. ${ }^{28}$ Figure 4 summarises an approach to treatment based on the severity of the airway disease. Because sarcoidosis is a multiorgan disease, treatment is usually targeted to the most severely affected system. The systemic treatment needed for other disease manifestations may directly benefit the upper airway.
However, local treatment for upper airway symptoms may still be useful. In some situations, patients with nasal and sinus SURT require only local measures, and saline irrigation and topical steroids may be sufficient to control disease. ${ }^{29} 30$ Other patients may require either systemic or topical antibiotics for superimposed infection.

While topical treatment may be helpful in selected cases, most case reports suggest that systemic treatment is usually required to control disease. Two institutions documenting treatment usage employed corticosteroids with steroid-sparing agents in $\geq 90 \%$ of their cases. ${ }^{13}$, The steroid-sparing agents varied between centres, with Aubart et al using antimalarial agents in $70 \%$ of cases, ${ }^{3}$ while Zeitlin et al used methotrexate in $72 \%$ of their patients. ${ }^{1}$ Other steroid-sparing agents included azathioprine, pentoxifylline, cyclophosphamide and thalidomide.

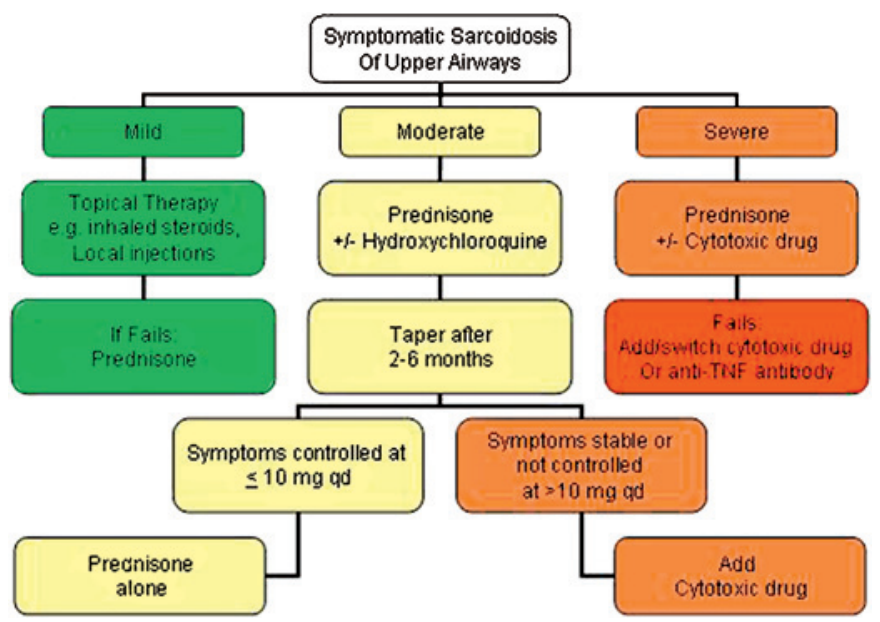

Figure 4 An approach to management of sarcoidosis of the upper respiratory tract. The severity of the disease is used to direct the approach to therapy. Surgical intervention may be necessary for 4 obstruction. qd, four times a day; TNF, tumour necrosis virus. 
A variety of systemic agents have been used for the treatment of SURT. Dosages, suggested monitoring and significant toxicities have been discussed in prior reports. ${ }^{28}$ Although there are limited data on specific agents in the treatment of SURT, some guidelines can be culled from the available literature.

The antimalarial agents can be effective in SURT. In the series by Aubart et al, hydroxychloroquine was used with prednisone in 12 of 14 cases. ${ }^{3}$ In the report from Zeitlin et al, all seven patients treated with antimalarial agents were also receiving another systemic treatment. ${ }^{1}$ However, Braun reported four SURT cases who failed to respond to hydroxychloroquine. ${ }^{4}$

Of the cytotoxic agents, methotrexate appears to be the most commonly prescribed steroid-sparing agent. ${ }^{13}$ This drug has been widely used for various manifestations of sarcoidosis, including pulmonary, cutaneous and neurological disease ${ }^{28}$ and it has been reported useful in destructive nasal disease. ${ }^{12}$ Azathioprine has also been prescribed for refractory cases. ${ }^{1}{ }^{3}{ }^{4}$ Although the cytotoxic agents mycophenylate and leflunomide have not been as widely studied, these drugs may represent alternatives for patients unable to tolerate either methotrexate or azathioprine.

Sinus disease is often noted in association with the chronic disease manifestation lupus pernio. ${ }^{31}$ The anti-tumour necrosis factor (TNF) antibodies infliximab and adalimumab can be effective in treating lupus pernio refractory to other treatments. ${ }^{28}$ In a single institution retrospective review of a large series of patients with sarcoidosis with lupus pernio, infliximab was superior to all other agents in controlling disease. ${ }^{32}$ Infliximab has also been shown to be superior to placebo in refractory pulmonary sarcoidosis.

Thalidomide is another agent useful in the treatment of lupus pernio. In one multicentre series, 14 patients with lupus pernio were treated with thalidomide in an open label, dose escalation trial. ${ }^{11}$ Four of the eight patients with sinus disease experienced improved sinus symptoms with thalidomide treatment.

Although anti-inflammatory treatment is usually necessary to control the disease, surgical intervention may be necessary to relieve obstruction. Surgery can improve the acute symptoms of obstruction and enhance sinus drainage, but it does not lead to long-term disease resolution. ${ }^{33}$ In one series, four of eight patients who underwent functional endoscopic sinus surgery or traditional sinus surgery were free of symptoms with long-term follow-up. ${ }^{1}$ However, all of these patients continued to receive systemic treatment for sarcoidosis.

Local treatment with intralesional steroid injections has been reported to be beneficial in laryngeal sarcoidosis. ${ }^{30}$ Although these intralesional injections may provide local disease improvement, laryngeal sarcoidosis is usually associated with other airway disease, ${ }^{6}$ which will necessitate additional treatment. However, the acute symptoms of upper airway obstruction may be relieved without requiring a tracheostomy. ${ }^{6}$ Although a single injection can be effective for only 3-6 months, this may provide sufficient time for systemic treatment, such as methotrexate, to become effective.

As noted earlier, bronchial stenosis can also complicate upper airway sarcoidosis, ${ }^{8}$ and systemic treatment may be beneficial in treating airway stenosis. The timing of systemic treatment may be important as earlier treatment seems to be associated with a better response. In one large series of airway sarcoidosis, Aubart et al noted that systemic treatment initiated within 3 months of diagnosis significantly improved overall outcome. ${ }^{3}$ While bronchoscopy is the diagnostic gold standard for obstruction, high resolution CT scanning can be useful for detecting airway obstruction. ${ }^{10}$

For non-responsive lesions, mechanical dilatation of the airways has been reported as useful in some cases. ${ }^{34}$ In one series of four refractory patients, all experienced disease improvement with rigid bronchoscopy and bougie dilatation; however, repeat dilatation was necessary in some cases. ${ }^{35}$ Because bronchial stenosis is often associated with postobstructive infection and bronchiectasis, ${ }^{835}$ treatment of infection may provide symptomatic relief.

\section{CONCLUSION}

SURT is an uncommon manifestation of the disease sarcoidosis. Because the granulomas of sarcoidosis can affect virtually any area of the upper airway, the presenting symptoms of SURT can range from sinus irritation, cough, hoarseness, dry eyes and mouth, to total airway obstruction. The differential diagnosis for this entity can also include Wegener granulomatosis as well as fungal diseases. SURT is frequently associated with the chronic facial lesion lupus pernio. Although local control measures with steroids, irrigation and surgery can be helpful, this entity represents a chronic form of the disease process which frequently requires systemic treatment with corticosteroids or other steroid-sparing agents including methotrexate, hydroxychloroquine, azathioprine or thalidomide. Future studies may demonstrate whether newer agents directed against TNF may be associated with a higher response rate. Because SURT can lead to airway obstruction, early systemic treatment may prevent irreversible narrowing.

\section{Competing interests None.}

Provenance and peer review Commissioned; not externally peer reviewed.

\section{REFERENCES}

1. Zeitlin JF, Tami TA, Baughman R, et al. Nasal and sinus manifestations of sarcoidosis. Am J Rhinol 2000;14:157-61.

2. Wilson R, Lund V, Sweatman M, et al. Upper respiratory tract involvement in sarcoidosis and its management. Eur Respir J 1988;1:269-72.

3. Aubart FC, Ouayoun M, Brauner M, et al. Sinonasal involvement in sarcoidosis: a case-control study of 20 patients. Medicine (Baltimore) 2006;85:365-71.

4. Braun JJ, Gentine A, Pauli G. Sinonasal sarcoidosis: review and report of fifteen cases. Laryngoscope 2004;114:1960-3.

5. Sims HS, Thakkar KH. Airway involvement and obstruction from granulomas in African-American patients with sarcoidosis. Respir Med 2007;101:2279-83.

6. Neville E, Mills RG, Jash DK, et al. Sarcoidosis of the upper respiratory tract and its association with lupus pernio. Thorax 1976;31:660-4.

7. Chambellan A, Turbie $\mathrm{P}$, Nunes $\mathrm{H}$, et al. Endoluminal stenosis of proximal bronchi in sarcoidosis: bronchoscopy, function, and evolution. Chest 2005;127:472-81

8. Udwadia ZF, Pilling JR, Jenkins PF, et al. Bronchoscopic and bronchographic findings in 12 patients with sarcoidosis and severe or progressive airways obstruction. Thorax 1990;45:272-5.

9. Shorr AF, Torrington KG, Hnatiuk OW. Endobronchial biopsy for sarcoidosis: a prospective study. Chest 2001;120:109-14.

10. Naccache JM, Lavole A, Nunes $\mathrm{H}$, et al. High-resolution computed tomographic imaging of airways in sarcoidosis patients with airflow obstruction. J Comput Assist Tomogr 2008;32:905-12.

11. Baughman RP, Judson MA, Teirstein AS, et al. Thalidomide for chronic sarcoidosis Chest 2002;122:227-32.

12. Hasni SA, Gruber BL. Sarcoidosis presenting as necrotizing sinus destruction mimicking Wegener's granulomatosis. J Rheumatol 2000;27:512-4.

13. Poate TW, Sharma R, Moutasim KA, et al. Orofacial presentations of sarcoidosis-a case series and review of the literature. Br Dent J 2008;205:437-42.

14. Baughman RP, du Bois RM, Lower EE. Sarcoidosis. Lancet 2003;361:1111-8.

15. deShazo RD, O'Brien MM, Justice WK, et al. Diagnostic criteria for sarcoidosis of the sinuses. J Allergy Clin Immunol 1999;103:789-95.

16. Slart RH, Jager PL, Poot L, et al. Clinical value of gallium-67 scintigraphy in assessment of disease activity in Wegener's granulomatosis. Ann Rheum Dis 2003;62:659-62.

17. Fuchs HA, Tanner SB. Granulomatous disorders of the nose and paranasal sinuses Curr Opin Otolaryngol Head Neck Surg 2009;17:23-7.

18. DeRemee RA. Sarcoidosis and Wegener's granulomatosis: a comparative analysis Sarcoidosis 1994:11:7-18.

19. Baum ED, Boudousquie AC, Li S, et al. Sarcoidosis with nasal obstruction and septal perforation. Ear Nose Throat J 1998;77:896-2

20. Bullen CL, Liesegang TJ, McDonald TJ, et al. Ocular complications of Wegener's granulomatosis. Ophthalmology 1983;90:279-90.

21. Comfere NI, Macaron NC, Gibson LE. Cutaneous manifestations of Wegener's granulomatosis: a clinicopathologic study of 17 patients and correlation to antineutrophil cytoplasmic antibody status. J Cutan Pathol 2007;34:739-47. 
22. Baughman RP, Shipley $R$, Desai $S$, et al. Changes in chest roentgenogram of sarcoidosis patients during a clinical trial of infliximab therapy: comparison of different methods of evaluation. Chest 2009;136:526-35.

23. Niimi H, Kang EY, Kwong JS, et al. CT of chronic infiltrative lung disease: prevalence of mediastinal lymphadenopathy. J Comput Assist Tomogr 1996;20:305-8.

24. Lower EE, Weiss KL. Neurosarcoidosis. Clin Chest Med 2008:29:475-92.

25. de Groot K, Schmidt DK, Arlt AC, et al. Standardized neurologic evaluations of 128 patients with Wegener granulomatosis. Arch Neurol 2001;58:1215-21.

26. Daum TE, Specks U, Colby TV, et al. Tracheobronchial involvement in Wegener's granulomatosis. Am J Respir Crit Care Med 1995;151:522-6.

27. Finkielman JD, Lee AS, Hummel AM, et al. ANCA are detectable in nearly all patients with active severe Wegener's granulomatosis. Am J Med 2007;120:643-14.

28. Baughman RP, Costabel U, du Bois RM. Treatment of sarcoidosis. Clin Chest Med 2008:29:533-48.
29. Krespi YP, Kuriloff DB, Aner M. Sarcoidosis of the sinonasal tract: a new staging system. Otolaryngol Head Neck Surg 1995;112:221-7.

30. Fergie N, Jones NS, Havlat MF. The nasal manifestations of sarcoidosis: a review and report of eight cases. J Laryngol Otol 1999;113:893-8.

31. Spiteri MA, Matthey F, Gordon T, et al. Lupus pernio: a clinico-radiological study of thirty-five cases. Br J Dermatol 1985:112:315-22.

32. Stagaki $\mathbf{E}$, Mountford WK, Lackland DT, et al. The treatment of lupus pernio: results of 116 treatment courses in 54 patients. Chest 2009;135:468-76.

33. Kay DJ, Har-El G. The role of endoscopic sinus surgery in chronic sinonasal sarcoidosis. Am J Rhinol 2001;15:249-54.

34. Fouty BW, Pomeranz M, Thigpen TP, et al. Dilatation of bronchial stenoses due to sarcoidosis using a flexible fiberoptic bronchoscope. Chest 1994;106:677-80.

35. Iles PB. Multiple bronchial stenoses: treatment by mechanical dilatation. Thorax 1981:36:784-6.

\section{Lung alert}

\section{Interferon gamma-1b does not improve survival for patients with idiopathic pulmonary fibrosis}

Idiopathic pulmonary fibrosis (IPF) is characterised by an insidious decline in pulmonary function, progressive worsening symptoms and death, with a median survival of 2-5 years from diagnosis. A methodologically limited meta-analysis has suggested that interferon $\gamma-1 b$ (IFN $\gamma$-1b) might improve survival.

To test this hypothesis, the INSPIRE study group conducted a double-blind trial, randomising 826 patients with IPF to receive IFN $\gamma-1 b$ or placebo. Patients had mild to moderately severe disease, with a forced vital capacity of $55-90 \%$ of the predictive value, a haemoglobin-corrected carbon monoxide transfer factor (TLCO) of 35-90\% of the predictive value, and a 6 -min walk distance (6MWD) of at least $150 \mathrm{~m}$. The primary end point was overall survival time from randomisation.

The study was stopped early because, at the second interim analysis, the hazard ratio for mortality in patients receiving IFN $\gamma$-1b showed an absence of minimum benefit compared with placebo. After a median duration of 64 weeks on treatment, $15 \%$ of patients on IFN $\gamma-1 \mathrm{~b}$ had died compared with $13 \%$ on placebo. Furthermore, IFN $\gamma$-1b did not significantly improve survival without lung transplantation, days without respiratory-related hospital admission, quality of life, $6 \mathrm{MWD}$, forced vital capacity or TLCO. Patients receiving IFN $\gamma$-1b reported more adverse events, but treatment adherence remained good.

This comprehensive study indicates that IFN $\gamma$-1b does not improve survival or confer any other significant benefit in patients with mild to moderate IPF, hence the strong recommendation by the joint ATS/ERS/JRS taskforce announced at the ERS meeting in September 2009 that IFN $\gamma$-1b should not be given to patients with IPF.

King TE Jr, Albera C, Bradford WZ, et al. Effect of interferon gamma-1b on survival in patients with idiopathic pulmonary fibrosis (INSPIRE): a multicentre, randomised, placebo-controlled trial. Lancet 2009;374:222-8.

\section{Alex Mackay}

Homerton University Hospital, Department of Respiratory Medicine, London, UK

Correspondence to Dr Alex Mackay, Specialist Registrar, Homerton University Hospital, Department of Respiratory Medicine, London, UK; malexmackay@hotmail.com

Competing interests None.

Provenance and peer review Not commissioned; not externally peer reviewed.

Thorax 2010;65:186. doi:10.1136/thx.2009.132886 